Proceedings

\title{
Fostering Renewable Energies and Energy Efficiency in the Water Sector Using PATs and Wheels ${ }^{\dagger}$
}

\author{
Helena M. Ramos ${ }^{1, *}$, Mariana Simão ${ }^{1}$, Aonghus McNabola ${ }^{2}$, Daniele Novara ${ }^{2}$ and \\ Armando Carravetta ${ }^{3}$ \\ 1 Civil Engineering, Architecture and Georesources Department, CERIS, Instituto Superior Técnico, \\ Universidade de Lisboa, 1049-001 Lisbon, Portugal; m.c.madeira.simao@tecnico.ulisboa.pt \\ 2 Department of Civil, Structural \& Environmental Engineering, Trinity College Dublin, the University of \\ Dublin, College Green, Dublin 2 D02 PN40, Ireland; amcnabol@tcd.ie (A.M.); novarad@tcd.ie (D.N.) \\ 3 Department of Civil, Architectural and Environmental Engineering, University of Naples "Federico II", \\ 80138 Naples, Italy; arcarrav@unina.it \\ * Correspondence: hramos.ist@gmail.com \\ + Presented at the 2nd International Research Conference on Sustainable Energy, Engineering, Materials and \\ Environment (IRCSEEME), Mieres, Spain, 25-27 September 2018.
}

Published: 7 November 2018

\begin{abstract}
REDAWN project will foster the adoption of hydropower energy recovery technology in built water networks in the Atlantic Area (AA). REDAWN will develop an adequate institutional, social and technological environment to foster greater resource efficiency in water networks. Pumps working as turbines (PAT) and other converters (wheels) became attractive to improve the water sector energy efficiency. However, the behavior of these devices are complex and it is difficult know its behavior. To overcome this problem, computational fluid dynamics (CFD) models were used in conjunction with the conceptualization and laboratory tests to explore its performance. Different modes were tested and simulated: single PAT mode and in parallel in pressurized pipe systems and a wheel in an open channel flow.
\end{abstract}

Keywords: REDAWN; pump as turbine (PAT); Venturi Flume; computational fluid dynamics (CFD); renewable energy; energy efficiency

\section{Introduction}

Reducing Energy Dependency in Atlantic area Water Networks (REDAWN) is a project which deals with the feasibility of hydropower in public water networks. The water industry is the 4th most energy intensive sector in the Atlantic Area (AA), responsible for significant contributions to climate change, and reductions in the competitiveness of the region due to the associated costs. REDAWN project will foster the adoption of hydropower energy recovery technology in built water networks in the Atlantic Area (AA). REDAWN will develop an adequate institutional, social and technological environment to foster greater resource efficiency in water networks.

At present significant potential exists to save energy, costs and environmental impacts in the AA water networks, but technological, institutional and social barriers prevent the exploitation of this resource. Flow energy is purposely wasted in water networks to restrict the levels of pressure, avoiding excessive leaking and bursting. This pressure control could also be achieved using turbines with the added benefit of energy production.

Water and Energy is a key problem for Europe where barriers include: (i) insufficient integration of water and energy, (ii) inadequate economic incentives to adopt efficient water-energy technologies and (iii) lack of low power technologies. 
The recover energy from water flowing in a transmission network to population centers are well accepted. However, in the distribution network, significant untapped potential exists for hydropower energy recovery not used yet.

REDAWN will also be the first project to explore the potential of all forms of water network for hydropower energy recovery (Figure 1).

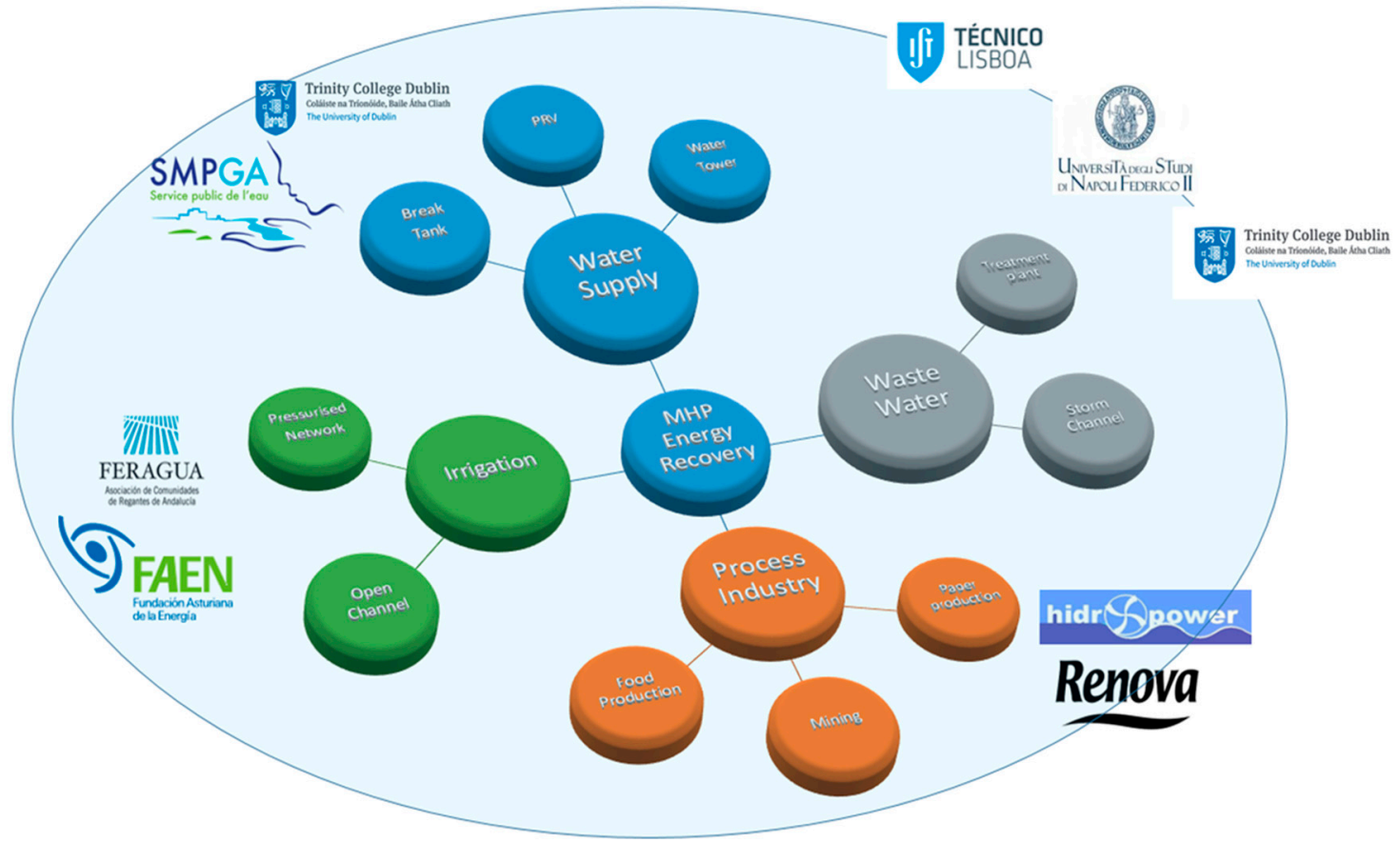

Figure 1. REDAWN project to foster energy recovery in the water networks.

Several investigations are under developed in the estimation of the potential energy available ij water systems, the pressure control associate to the leakage, the waste of flow energy in different mechanical devices, specifications of characteristic curves of low-cost energy converters, flow characterization, and performance estimation for pumps as turbines.

For water distribution networks (i.e., low power available less than $100 \mathrm{~kW}$ ) pumps working as turbines (PATs) can be seen as an interesting alternative solution to reduce the pressure in pipe systems, replacing or in conjunction with pressure reduction valves. This renewable system, which presents feasibility indexes with low payback periods ( $<5$ years), can lead to the improvement of the future water systems sustainability [1].

Several investigations on PATs and on Wheels domain are under study for the flow behavior and the influence in the operation points, due to the turbulence occurrence with vortex formation [24]. These flow structures create difficulties in the use of affinity laws to extrapolate other operating conditions, which need to be validated by detailed experimental data [5-11].

Hence, an experimental set-up was established at the CERIS-Hydraulic Lab of Instituto Superior Técnico at the University of Lisbon and used for numerical validation. All required parameters were measured in a single PAT for a more constant flow conditions and 2 PATs in parallel when the system imposes more regulation of the flow.

\section{Materials and Methods}

\subsection{Experimental Tests and CFD in PATS}

The PAT facility system allows the study the PAT performance under different steady flows and operating conditions. The PAT used is an Etarnom 32-125 from KSB, with the best efficiency point (BEP) at $3.60 \mathrm{~L} / \mathrm{s}$ and $4.43 \mathrm{~m}$ w.c. The specific speed of the machine is $51 \mathrm{rpm}(\mathrm{m}, \mathrm{kW})$ and its nominal rotational speed is $1020 \mathrm{rpm}$. Figure 2 shows the two experimental set-up related to the two 
different PAT operating conditions. The experiments were carried out for different rotational speeds (1020, 1275 and $1500 \mathrm{rpm})$ and different flows (2.9-5.2 L/s) in steady state conditions. The pressure was recorded in two pressure transducers located upstream and downstream of each PAT set-up. The rotational speed was measured using a digital tachometer. Figure 3 shows the experimental characteristic curves of a single PAT and for the 2 PATs in parallel with a different flow distribution and rotational speeds.

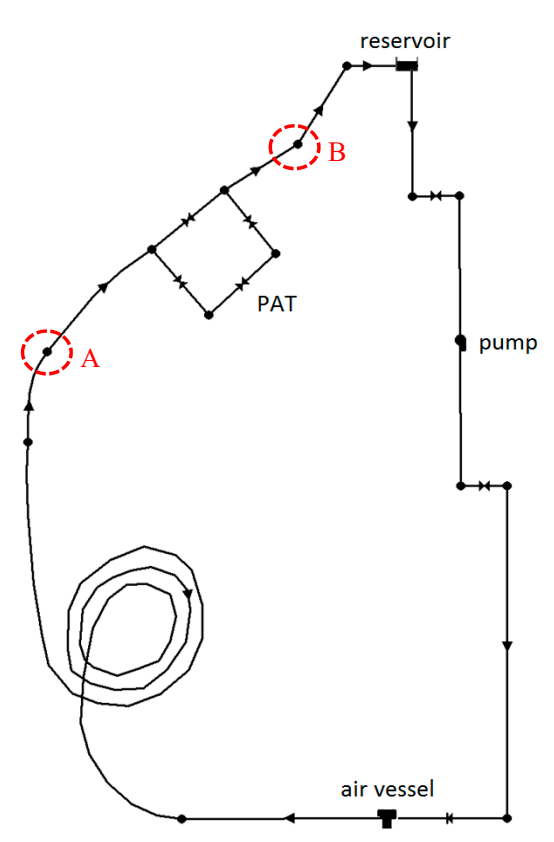

(a)

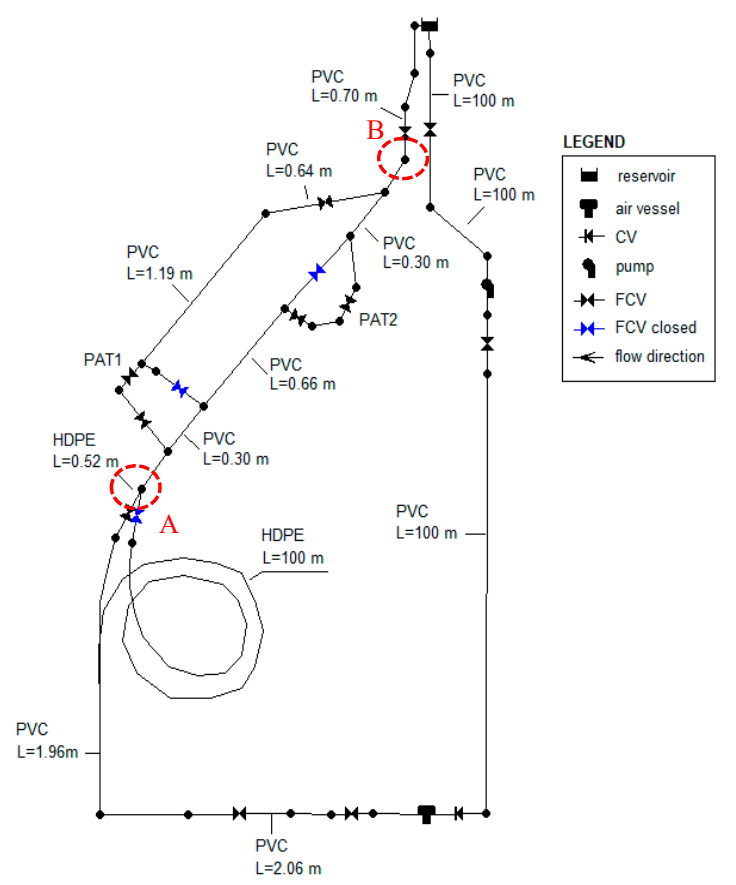

(b)

Figure 2. Scheme of the experimental hydraulic facility at CERIS-IST Lab: (a) single PAT; (b) two identical PATs in parallel.

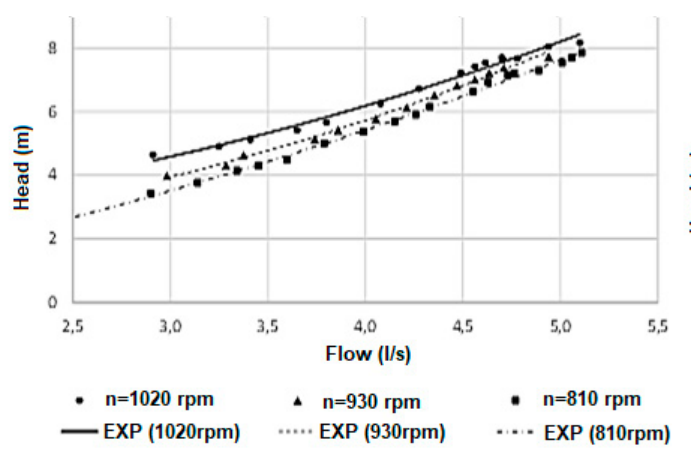

(a)

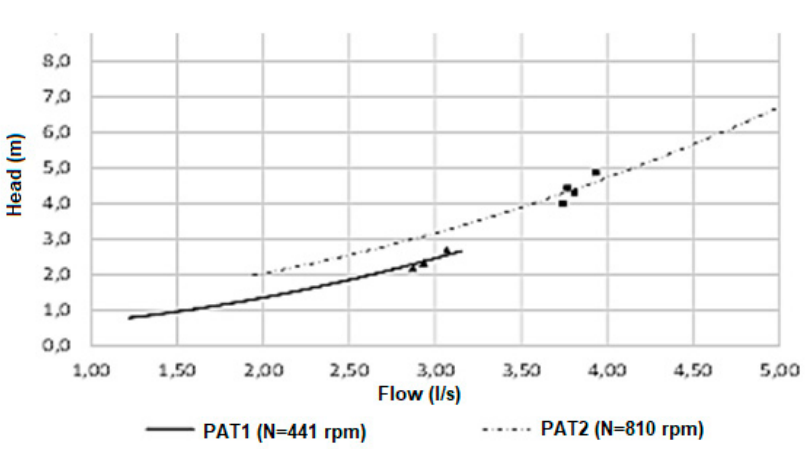

(b)

Figure 3. Characteristic curves: (a) single PAT; (b) PATs in parallel (with different flows).

\subsection{Open Channel in a WWTP and CFD in Wheels}

Possibility of installing in a typical exit open channel of a Waste Water Treatment Plant (WWTP), a hydrokinetic turbine at correspondence of Venturi Flume, or a hydrostatic wheel machine after the narrow section (Figure 4 top) are investigated. The theory behind a hydrostatic pressure wheel is based on the hydrostatic law (Figure 4 bottom), while the theoretical available power corresponds to:

$$
P_{a v .}=\rho g Q\left(h_{1}-h_{2}\right)
$$



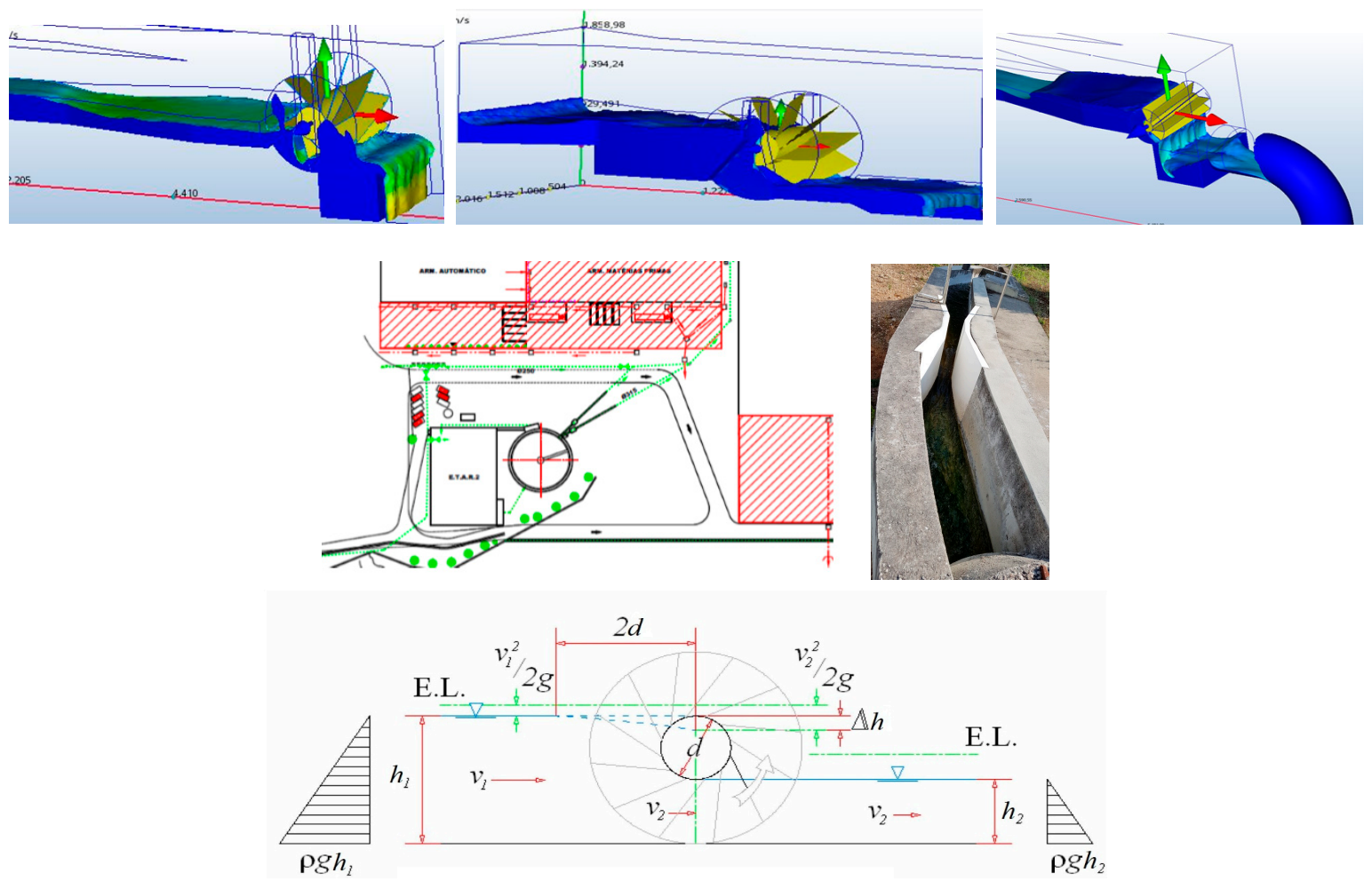

Figure 4. Different configurations and an ideal hydrostatic wheel machine for WWTPs [11].

\section{Results}

\subsection{PATS}

The boundary conditions assigned to sections A and B (Figure 2), are the inlet and outlet measured points of each PAT system, i.e., the volume of the flow rate, corresponding to each test, was allocated to the inlet condition, as for the outlet condition, the pressure recorded in the pressure transducer (located downstream of each set-up) was set. For the rotation of the impellers, the corresponding rotational speeds obtained experimentally were also considered (Figures 5). The centrifugal force effect rises with the increasing of the rotational speed. Figure 6 shows the pressure distribution of the PAT for $810 \mathrm{rpm}$ (single mode) and $440 \mathrm{rpm}$ and 810 (for PAT1 and PAT2 in parallel).

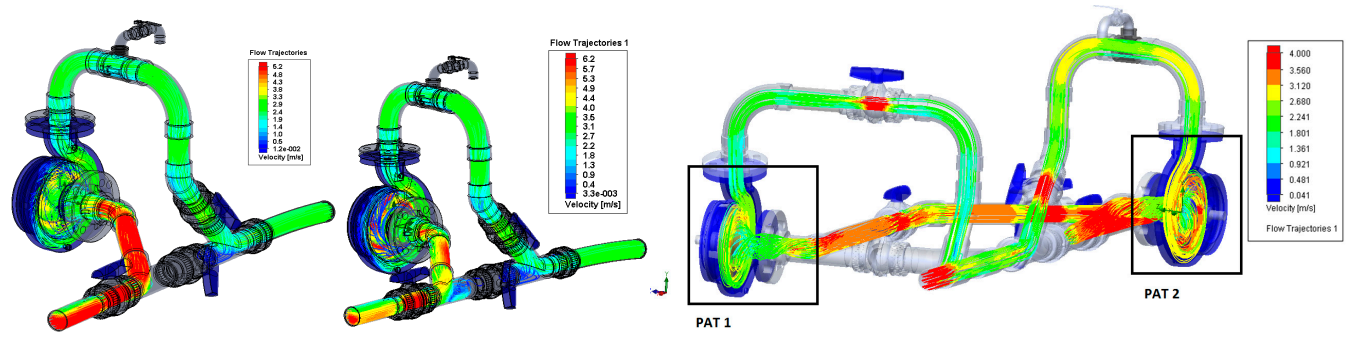

Figure 5. Velocity streamlines for single mode operation (a) $\mathrm{N}=810 \mathrm{rpm}$; (b) $\mathrm{N}=1020 \mathrm{rpm}$; and (c) for parallel mode operation with NPAT1 $=440 \mathrm{rpm}$ and NPAT2 $=810 \mathrm{rpm}$. 


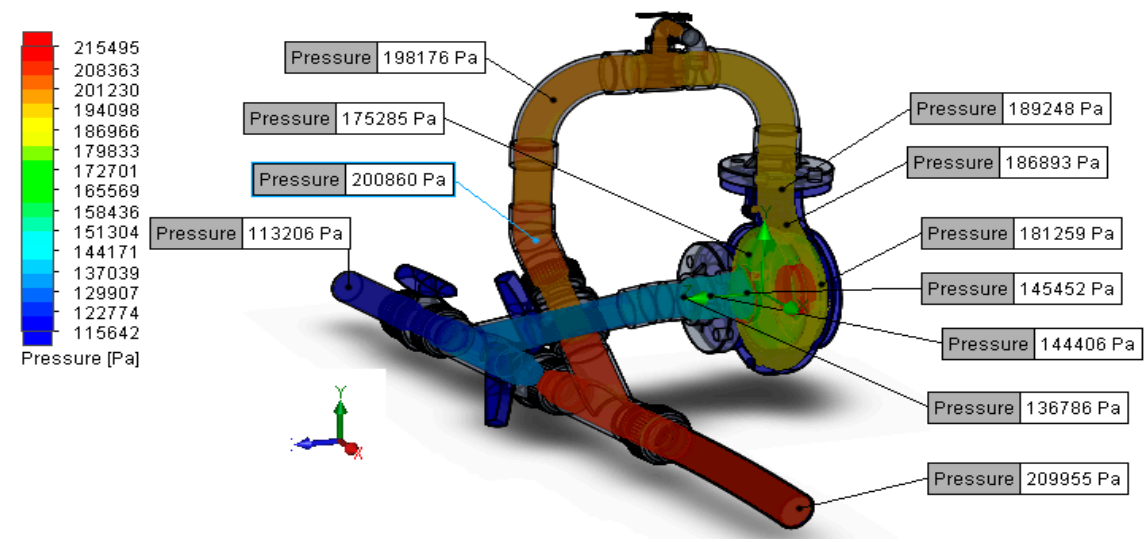

(a)

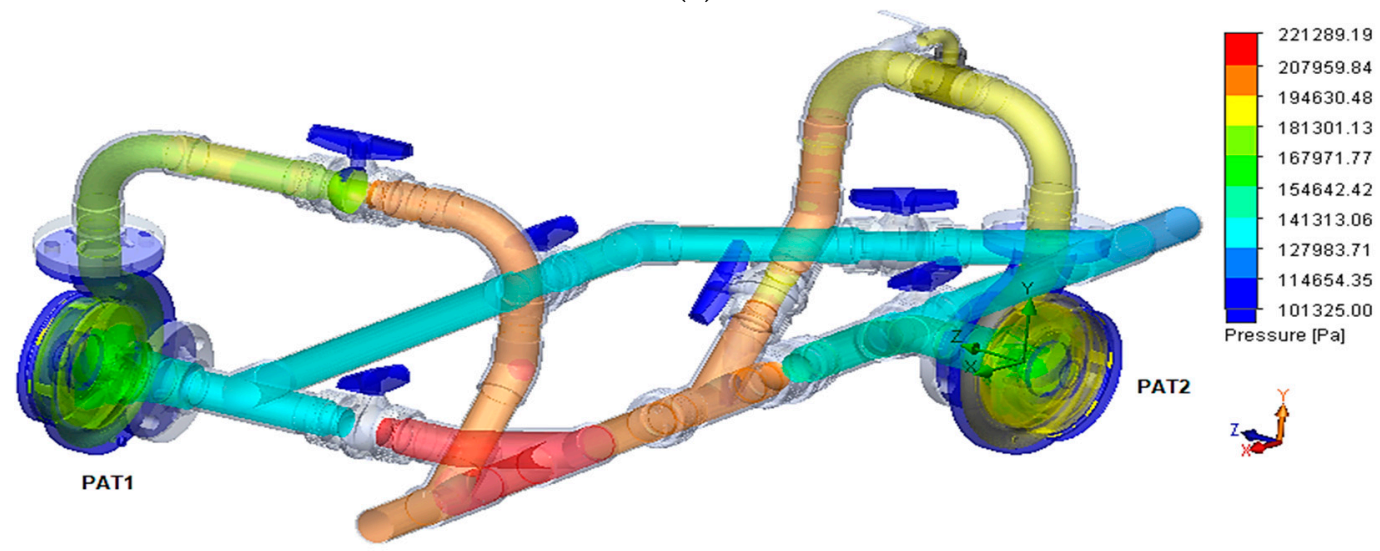

(b)

Figure 6. Pressure contours: (a) single PAT; (b) PATs in parallel.

Comparing the results from single mode and in parallel operation, in the latter one it is possible to run the system to cover different flow rates than using only a single PAT (Table 1). When working a single PAT, the rotational speed and the efficiency are higher for a high flow rate. Figure 7 shows the characteristic curves achieved for both conditions, after validating the CFD model $[6,10]$.

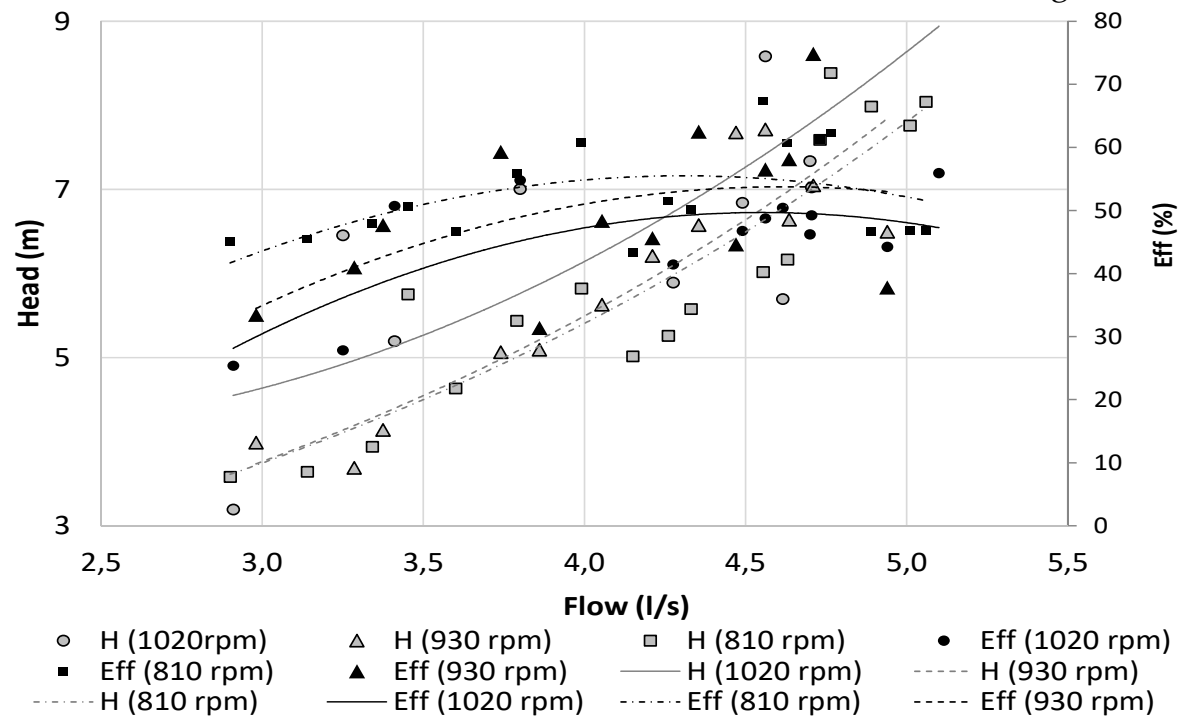

(a) 


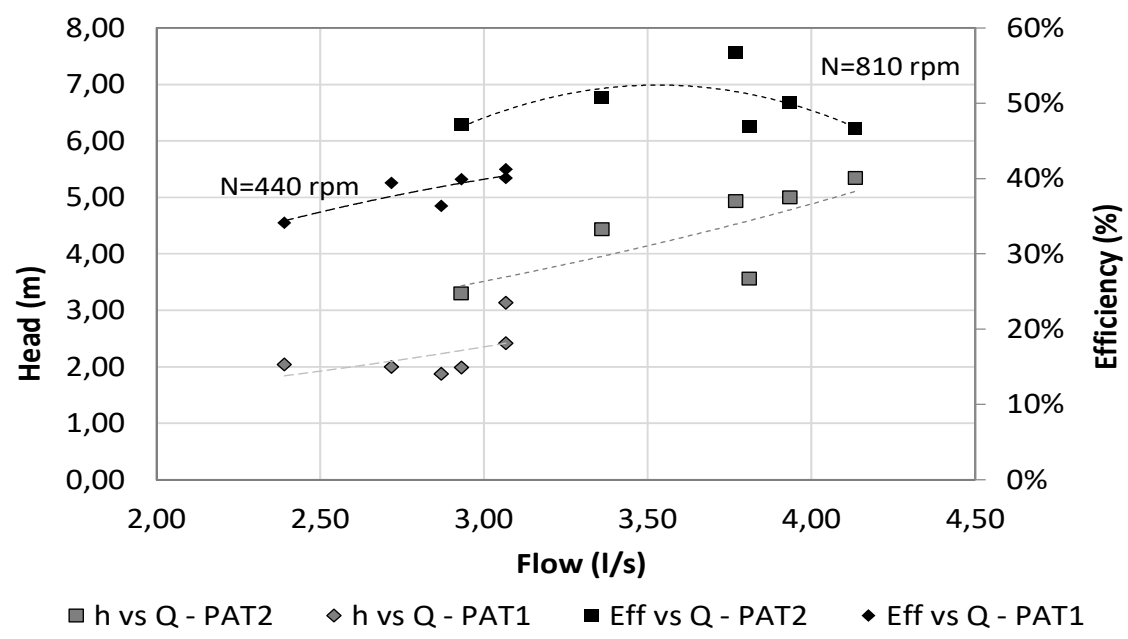

(b)

Figure 7. Characteristic curves: (a) single PAT for different rotational speeds; (b) PATs in parallel for 440 and $810 \mathrm{rpm}$ in each PAT.

Table 1. Operation range of experimental set-ups (in single and in parallel operation).

\begin{tabular}{ccccc}
\hline Set-Up & Flow Range (L/s) & Head Range (m) & Efficiency (\%) & N Range (rpm) \\
\hline Single mode & $3-5$ & $3-8$ & $30-60$ & $800-1020$ \\
\hline Parallel mode & $\begin{array}{c}2.3-4.3 \text { in each PAT } \\
\left(Q_{\text {total }}=6.6 \mathrm{~L} / \mathrm{s}\right)\end{array}$ & $2-6$ & $35-60$ & $440-810$ \\
\hline
\end{tabular}

In the open channel system some simulations of the hydrostatic wheel were presented after CFD simulations (Figure 8). The most promising technology for open channels can be selected as the Hydrostatic Wheel Machine (HWM) which is specifically designed for low heads.
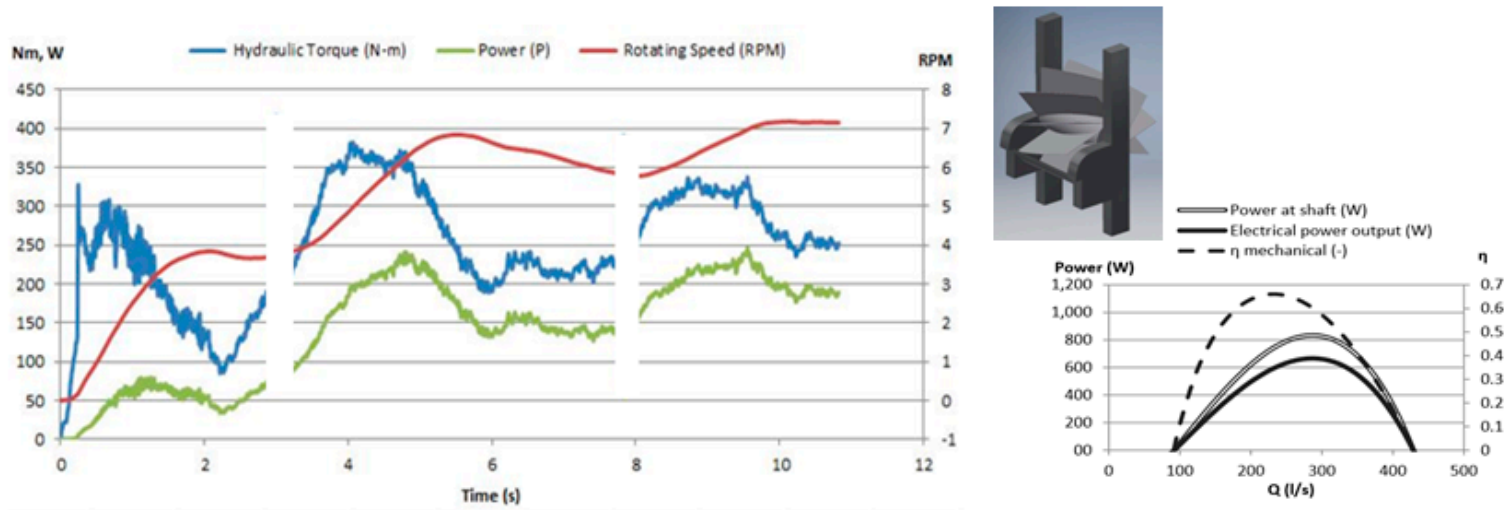

Figure 8. Variation of mechanical and electrical parameters of a hydrostatic wheel machine [11].

\section{Conclusions}

Water distribution networks (WDNs) appear as a new opportunity to generate clean energy using pumps working as turbines (PATs) instead of energy dissipaters by pressure reduction valves (PRVs). Experimental and CFD simulations were performed for detailed flow analysis to study the velocity variation in a single PAT with the specific speed of $21 \mathrm{rpm}\left(\mathrm{m}, \mathrm{m}^{3} / \mathrm{s}\right)$ and in 2 PATs in parallel. The obtained experimental data and CFD results allowed to better understand the flow behavior through the PAT, and the analysis of the velocity vectors for different operating conditions associated to each flow discharge, head and rotational speed. Different rotational speeds were imposed in each PAT system associated to each operating mode. The benefits associated to the PATs working in parallel are the possibility to cover a higher flow range variation with best efficiency 
conditions than the one for a single PAT. Despite a significant and guaranteed daily water flow passing through a WWTP outlet channel, the low available head allows a hypothetical application of HWM installation to perform generated electricity and economic attractiveness.

In particular, discounted payback period has been evaluated as equal to 2 and 8 years within the most optimistic scenarios for PATs and water wheels, respectively and maximum values of 4 and 15 years for the worst configuration parameters, for both analyzed machines.

\section{References}

1. Carravetta, A.; Del Giudice, G.; Fecarotta, O.; Ramos, H. PAT design strategy for energy recovery in water distribution networks by electrical regulation. Energies 2013, 6, 411-424, doi:10.3390/en6010411.

2. Yang, S.S.; Derakhshan, S.; Kong, F.Y. Theoretical, numerical and experimental prediction of pump as turbine performance. Renew. Energy 2012, 48, 507-513.

3. Ramos, H.; Almeida, A.B. Parametric Analysis of WaterHammer Effects in Small Hydro Schemes. J. Hydraul. Eng. 2022, 128, 689-696.

4. Simão, M.; Ramos, H.M. Hydrodynamic and performance of low power turbines: Conception, modelling and experimental tests. Int. J. Energy Environ. 2010, 1, 431-444.

5. Darmawi, R.; Sipahutar, S.; Bernas, M.; Imanuddin, S. Renewable energy and hydropower utilization tendency worldwide. Renew. Sustain. Energy Rev. 2013, 17, 213-215.

6. Pérez-Sánchez, M.; Sánchez-Romero, F.; Ramos, H.; López-Jiménez, P. Energy recovery in existing water networks: Towards greater sustainability. Water 2017, 9, 97, doi:10.3390/w9020097.

7. Simão, M.; Pérez-Sánchez, M.; Carravetta, A.; López-Jiménez, P.; Ramos, H.M. Velocities in a Centrifugal PAT Operation: Experiments and CFD Analyses. Fluids 2017, 3, 3.

8. Carravetta, A.; Derakhshan, H.S.; Ramos, H.M. Pumps as Turbines, Fundamentals and Applications; Springer International Publishing: Berlin, Germany, 2017; ISBN 978-3-319-67506-0, ISBN 978-3-319-67507-7 (eBook), doi:10.1007/978-3-319-67507-72018.

9. Ramos, H. Guidelines for Design of Small Hydropower Plants; WREAN (Western Regional Energy Agency and Network) and DED (Department of Economic Development-Energy Division): Belfast, UK, 2000; ISBN 972-96346-4-5.

10. Pérez-Sánchez, M.; Simão, M.; López-Jiménez, P.; Ramos, H.M. CFD Analyses and Experiments in a PAT Modeling: Pressure Variation and System Efficiency. Fluids 2017, 2, 51.

11. Novara, D. Energy Harvesting from Municipal Water Management Systems: From Storage and Distribution to Wastewater Treatment. Master's Thesis, Institute of Science and Technology Austria, Klosterneuburg, Austria, 2016.

(C) 2018 by the authors. Licensee MDPI, Basel, Switzerland. This article is an open access article distributed under the terms and conditions of the Creative Commons Attribution (CC BY) license (http://creativecommons.org/licenses/by/4.0/). 